Article

\title{
Quantitative Structural Analysis of Polystyrene Nanoparticles Using Synchrotron X-ray Scattering and Dynamic Light Scattering
}

\author{
Jia Chyi Wong ${ }^{1,2}$, Li Xiang ${ }^{2}$, Kuan Hoon Ngoi ${ }^{1,2}$, Chin Hua Chia ${ }^{1, * \mathbb{D}}$, Kyeong Sik Jin ${ }^{3, *}$ and \\ Moonhor Ree ${ }^{2, *}$ \\ 1 Materials Science Program, School of Applied Physics, Faculty of Science and Technology, \\ Universiti Kebangsaan Malaysia, Bangi 43600, Malaysia; wongjiachyi@gmail.com (J.C.W.); \\ ngoikuanhoon@gmail.com (K.H.N.) \\ 2 Department of Chemistry, Polymer Research Institute, and Pohang Accelerator Laboratory, \\ Pohang University of Science and Technology, Pohang 37673, Korea; lea1990@postech.ac.kr \\ 3 Pohang Accelerator Laboratory, Pohang University of Science \& Technology, Pohang 37673, Korea \\ * Correspondence: chia@ukm.edu.my (C.H.C.); jinks@postech.ac.kr (K.S.J.); ree@postech.ac.kr (M.R.)
}

Received: 1 February 2020; Accepted: 18 February 2020; Published: 19 February 2020

\begin{abstract}
A series of polystyrene nanoparticles (PS-1, PS-2, PS-3, and PS-4) in aqueous solutions were investigated in terms of morphological structure, size, and size distribution. Synchrotron small-angle X-ray scattering analysis (SAXS) was carried out, providing morphology details, size and size distribution on the particles. PS-1, PS-2, and PS-3 were confirmed to behave two-phase (core and shell) spherical shapes, whereas PS-4 exhibited a single-phase spherical shape. They all revealed very narrow unimodal size distributions. The structural parameter details including radial density profile were determined. In addition, the presence of surfactant molecules and their assemblies were detected for all particle solutions, which could originate from their surfactant-assisted emulsion polymerizations. In addition, dynamic light scattering (DLS) analysis was performed, finding only meaningful hydrodynamic size and intensity-weighted mean size information on the individual PS solutions because of the particles' spherical nature. In contrast, the size distributions were extracted unrealistically too broad, and the volume- and number-weighted mean sizes were too small, therefore inappropriate to describe the particle systems. Furthermore, the DLS analysis could not detect completely the surfactant and their assemblies present in the particle solutions. Overall, the quantitative SAXS analysis confirmed that the individual PS particle systems were successfully prepared with spherical shape in a very narrow unimodal size distribution.
\end{abstract}

Keywords: polystyrene nanoparticles; synchrotron X-ray scattering analysis; dynamic light scattering analysis; particle morphology; particle shape; particle size; size distribution; radial density profile

\section{Introduction}

Nanoparticles are accepted widely as materials of several hundred nanometers or less in geometrical dimension. They are commonly thought to have a most simple shape-e.g., spherical form-among nanomaterials. However, they reveal a number of different geometries in detail and a variety of density profiles from the center to the surface. Nevertheless, due to the merits based on nanoscale dimensions, nanoparticles have led to emerging applications in various fields, such as smart coatings, coating additives, polymers, agrochemicals, detergents, lubricants, cutting oils, corrosion inhibitors, catalysts, chemical storage, environmental remediation, energy generation and storage, electro-optics, photonics, microelectronics, cosmetics, pharmaceutics, biosensors, medical diagnostics, medical therapy, drug delivery carriers, foods, and so on [1-14]. 
For a material, all properties are generally known to depend upon the morphological structure and molecular characteristics. In the case of nanoparticles, the properties and performance are also highly dependent upon the morphology details as well as the chemical characteristics [1,7-14]. The morphology details include three-dimensional geometry, surface texture, size and size distribution, surface-to-volume ratio, and density gradient profile. In particular, particle size is known as a crucial factor to govern the properties and performance [13-20]. To measure nanoparticle size, dynamic light scattering is currently used most widely because of the experimental simplicity and automatized data analysis scheme using the instruments developed in compact type [21-26]. However, dynamic light scattering very often provides unrealistically large size and distribution [27-34]. Electron microscopy is also widely used to characterize nanoparticles, but always requires specific measurement conditions such as dried or freeze samples and vacuum environment [35-37]. As an alternative of electron microscopy, atomic force microscopic analysis is applicable for nanoparticles in aqueous medium or another solvent medium [28,38-41]. There are several other methods available, including X-ray scattering, static light scattering, laser diffraction, neutron scattering, fluorescence correlation spectroscopy, sedimentation, sieving, optical particle counting, electrozone sensing, and resistive pulse sensing [42-56]. However, the individual methods have some disadvantages including detection limit and valid boundary condition, leading to differences even in the size of nanoparticle $[28,32,33,40,43,49,50,53-56]$. Furthermore, they often face difficulties in analyzing measured data mathematically in a quantitative manner. The correct interpretation and understanding of analysis results in could also be challenging because of the different underlying metrological theories, concepts, and boundary conditions. Therefore, nanoparticles still need urgent advances even in the size and size distribution measurement with higher precision and higher accuracy. Their structural details (geometrical shape, surface texture, density gradient profile, etc.) are further analyzed for better understanding and appropriate utilization.

In this study, we have attempted to investigate a series of polystyrene (PS) nanoparticles in various sizes in order to get insights into their morphological structure and size distribution. For this investigation, synchrotron small-angle X-ray scattering (SAXS) technique has been chosen because of its ability to determine structure and size details and the availability of synchrotron radiation X-ray source and experimental beamline facility. Dynamic light scattering (DLS) method has been additionally chosen due to its availability in an economic way as a compact and handy instrument and the easy usage with a built-in automatized data analysis software package. The quantitative SAXS analysis provides geometrical shape, size, size distribution, and density gradient profile. The DLS gives additional information on the particle size and distribution.

\section{Materials and Methods}

A series of PS nanoparticles were received from Thermo Fisher Scientific Company (Seoul, Korea): PS-1 (Nanosphere ${ }^{\mathrm{TM}} 3080 \mathrm{~A}, 1 \mathrm{wt} \%$ in aqueous solution), PS-2 (Nanosphere ${ }^{\mathrm{TM}} 3050 \mathrm{~A}, 1 \mathrm{wt} \%$ in aqueous solution), PS-3 (Nanosphere ${ }^{\mathrm{TM}} 3030 \mathrm{~A}, 1 \mathrm{wt} \%$ in aqueous solution), and PS-4 (Nanosphere ${ }^{\mathrm{TM}} 3020 \mathrm{~A}$, $1 \mathrm{wt} \%$ solid in aqueous solution). For each sample solution, a part was taken out, put into a centrifuge tube, and followed by centrifugation at $12,000 \mathrm{rpm}$ for $32 \mathrm{~h}$ in a refrigerated centrifuge (model 1248R, LABOGENE, Seoul, Korea) at $25^{\circ} \mathrm{C}$. Then, the upper liquid layer was carefully pipetted out from the centrifuge tube without touching the sedimented nanoparticles, put into a vial, and kept as a supernatant for $X$-ray scattering measurements.

X-ray scattering measurements were conducted at the $4 \mathrm{C}$ beamline $[32,44,45,57]$ of the PLS-II facility (third-generation synchrotron facility of $3.0 \mathrm{GeV}$ power), Pohang Accelerator Laboratory (Pohang, Korea). The X-ray beam with a wavelength $\lambda$ of $0.07336 \mathrm{~nm}$ was used. A two-dimensional (2D) charged-coupled detector (CCD: model Rayonix 2D SX 165, Evanston, IL, USA) was employed. Quartz capillary cells with $1.5 \mathrm{~mm}$ outer diameter were used. The sample-to-detector distances (SDD) of $1.0 \mathrm{~m}$ and $4.0 \mathrm{~m}$ were chosen. The data collection time was $30 \mathrm{~s}$. The scattering angles were calibrated by using a precalibrated Ti-SBA-15 (Sigma-Aldrich Company, Seoul, Korea) and silver 
behenate (Tokyo Chemical Industry (TCI), Tokyo, Japan) as standards. The individual 2D scattering data were circularly averaged with respect to the X-ray beam center and then followed by normalizing to the transmitted X-ray beam intensity which was monitored with a scintillation counter positioned behind the sample. The scattering data was further corrected for the scattering arising from either the water or the supernatant.

DLS measurements were carried out at $25.0^{\circ} \mathrm{C}\left( \pm 0.1^{\circ} \mathrm{C}\right)$ by using a Malvern DLS instrument (model Zetasizer Nano ZS90, Malvern Instruments Ltd., Worcestershire, UK) equipped with a He-Ne laser source of $632.8 \mathrm{~nm}$ wavelength; a detector was set at a scattering angle of $90^{\circ}$. Low-volume quartz batch cuvettes (model ZEN2112, Malvern Instruments Ltd., Worcestershire, UK) were employed as sample cells.

\section{Results and Discussion}

\subsection{PS-1}

Figure 1a presents a representative of the synchrotron X-ray scattering data of the PS-1 particle in aqueous solution measured at room temperature and corrected for the water medium. The scattering profile has been attempted to be analyzed in a qualitative manner by using the indirect Fourier transformation (IFT) analysis [58], which is a model independent scattering analysis method; the IFT method detail is given in Supporting Information. The obtained $p(r)$ profile is shown in Figure $1 \mathrm{~b}$, providing structural information: (i) $R_{\mathrm{g}, \mathrm{IFT}}=33.8 \mathrm{~nm}$ (radius of gyration), (ii) $R_{\mathrm{max}, \mathrm{IFT}}=45.5 \mathrm{~nm}$ (radius at the peak maximum), and (iii) $D_{\text {max,IFT }}=87.5 \mathrm{~nm}$ (maximum dimension) (Table 1 ). The $p(r)$ profile is apparently close to a bell curve that is commonly observed for an ideal sphere. $R_{\max , \mathrm{IFT}} / R_{\mathrm{g}, \mathrm{IFT}}=1.35$, which is close to 1.36 for an ideally homogenous sphere [4,59]. $D_{\max , \mathrm{IFT}} / R_{\max , \mathrm{IFT}}=1.92$, which is close to 2 for an ideal sphere. These results collectively inform that PS-1 is a sphere-like nanoparticle revealing a certain size, namely $R_{\mathrm{g}, \mathrm{IFT}}=33.8 \mathrm{~nm}$ and $R_{\mathrm{max}, \mathrm{IFT}}=45.5 \mathrm{~nm}$.
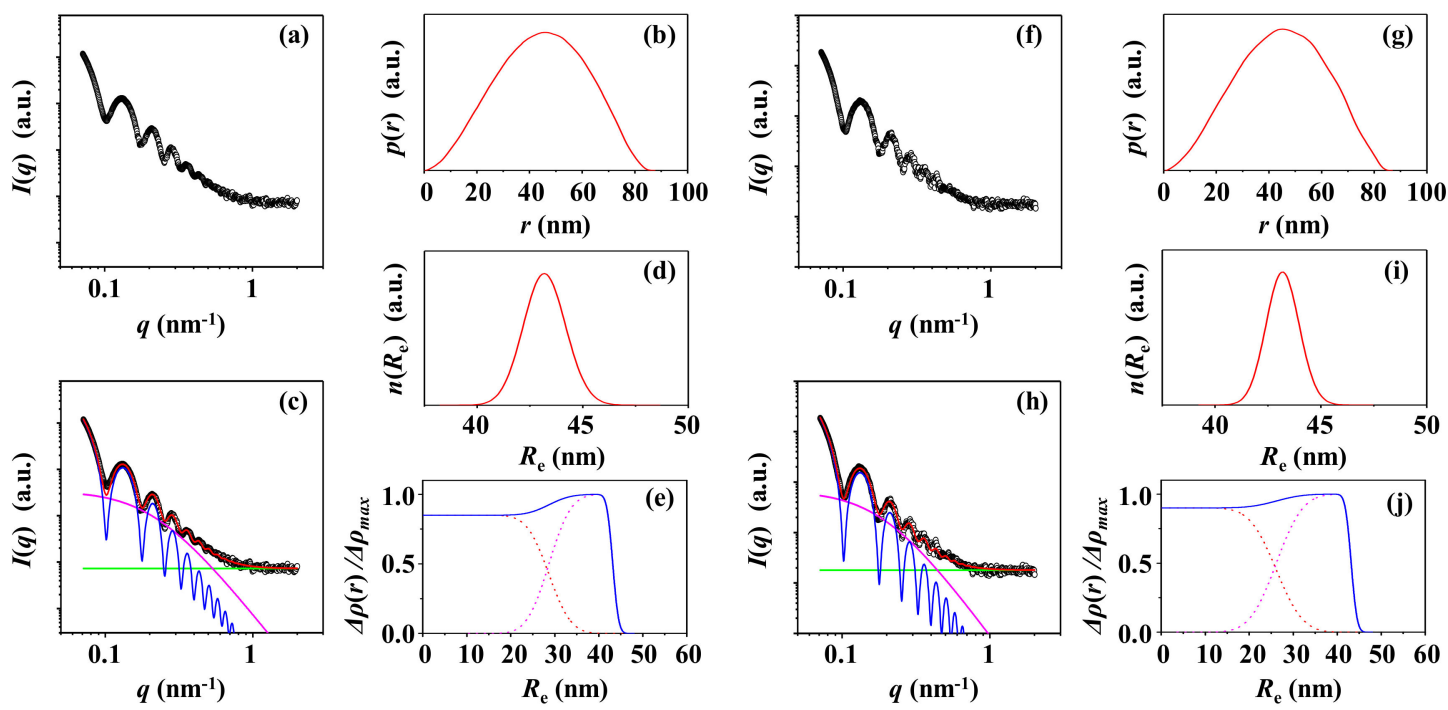

Figure 1. X-ray scattering analysis of PS-1. (a) SAXS profile measured at room temperature and corrected for water as the solution medium: (b) pair distance distribution functions $p(r)$ obtained from the scattering profile using the IFT method; (c) data analysis results; (d) radius distribution obtained by the data analysis in (c); (e) density distribution obtained by the data analysis in (c). (f) SAXS profile measured at room temperature and corrected for the supernatant as a solution medium: (g) pair distance distribution functions $p(r)$ obtained from the scattering profile using the IFT method; (h) data analysis results; (i) radius distribution obtained by the data analysis in (h); (j) density distribution obtained by the data analysis in $(\mathbf{h}) .(\mathbf{c}, \mathbf{h})$ the open symbols are the measured data and the red solid line represents the sum of the profiles obtained by fitting the data using two-phase ellipsoid model (blue line) and local random two-phase contributions (purple line) and the background (green line). 
With the IFT analysis results, the X-ray scattering data have been further subjected to quantitative structural model analysis. Various structural models have been considered and tested. As a result, a two-phase ellipsoidal model approach is found most suitable. As shown in Figure 1c, the scattering data are well fitted by using the two-phase ellipsoidal model combined with the local density fluctuations based on random two phases (see Figure 1c); the scattering formulas are given in Supporting Information. This analysis provides the nanoparticle shape and structural parameter details (Table 1). The ellipsoidicity ratio $\varepsilon[=$ (polar radius)/(equatorial radius)] is determined to be 1.0, confirming that PS-1 is spherical. The radius of gyration $R_{\mathrm{g}}$ is $33.5 \mathrm{~nm}$, which is very close to that determined by the IFT analysis. The mean radius $R_{\mathrm{e}}$ is $43.2 \mathrm{~nm}$, which consists of a core radius $r_{\mathrm{c}}$ of $29.2 \mathrm{~nm}$, and a shell thickness $t_{\mathrm{s}}$ of $14.0 \mathrm{~nm}$. In the whole particle, the average correlation length $\xi$ of local density fluctuations (i.e., random two phases) is additionally estimated to be $4.6 \mathrm{~nm}$. These local density fluctuations may originate mainly from the chemical crosslinks formed in the PS-1 synthesized by using emulsion polymerization technique. The radius (i.e., size) distribution of PS-1 particles is found to follow the Schultz-Zimm function [60]; the obtained radius distribution is presented in Figure $1 \mathrm{~d}$.

The radial electron density distribution profile $\Delta \rho(r)$ is one of the interesting structural parameters for understanding the PS-1 particle. Thus, the density profile has been first attempted to be extracted from the $p(\mathrm{r})$ profile obtained from the IFT analysis. However, this extraction approach could give unrealistic density profiles as multiple solutions. This failure may be attributed to the IFT software's limitations including the assumption of monodispersity in size distribution. Instead, the numerical Fourier transformation of the respective scattering amplitudes determined in the quantitative structural model analysis is found to provide a more realistic density profile, as shown in Figure 1e; the analysis detail is in Supporting Information.

This quantitative analysis has been extended to extract $p(r)$ profile. Namely, with the structural parameters determined by the quantitative model analysis, a scattering profile has been reconstructed and then Fourier-transformed, giving $p(r)$ profile (Figure S1 in Supplementary Materials). From the $p(r)$ profile, a set of structural parameters $\left(R_{\mathrm{g}}, R_{\max }\right.$, and $\left.D_{\max }\right)$ has been obtained, as listed in Table S1. Overall, the extracted $p(r)$ profile and resulting structural parameters are reasonably well matched with those determined directly from the measured scattering data by the IFT analysis. This crosscheck again confirms the validity of the density profile extracted above and furthermore the scattering data analysis done successfully.

It was informed from the supplier that the aqueous PS-1 solution includes a small quantity of surfactant which was employed in the particle preparation by surfactant-based emulsion polymerization; such surfactant is known to further stabilize the dispersion of the resulting particles in the solution. The surfactant residue may also cause scattering with X-ray beam and influence the X-ray scattering data arising for the PS-1 solution. Thus, the PS-1 solution has been separated into the PS-1 particles and the supernatant by centrifugation. For the obtained supernatant, X-ray scattering measurement has been carried out and then used to correct the X-ray scattering data measured for the PS-1 solution. The corrected scattering data are presented in Figure $1 \mathrm{f}$ and then followed by IFT and structural model analyses. The analysis results are shown in Figure $1 \mathrm{~h}-\mathrm{j}$. The obtained structural parameters are almost the same as those determined by analyzing the $X$-ray scattering data corrected with the water medium (Table 1 ). These analyses collectively confirm that the presence of the surfactant molecules in the solution causes no positive or negative contributions on the X-ray scattering data of the PS-1 solution. This result may be attributed to large size differences between the PS-1 particles and the surfactant molecules including their possible assemblies and possibly low concentration of the surfactant in the solution.

The PS-1 solution has been additionally examined by DLS. Figure 2a-1 shows a representative second order autocorrelation function $g_{2}(q, t)$ constructed from the fluctuating scattered intensity $I_{\mathrm{s}}(q, t)$ data measured at a fixed scattering angle of $90^{\circ}$ as a function of time. From the $g_{2}(q, t)$ profile, the first order autocorrelation function $g_{1}(q, t)$ (i.e., field autocorrelation function) has been extracted by using the Siegert relation $[32,62,63]$ and then analyzed using the Malvern Zetasizer analysis software package based on the cumulant method and the non-negatively constrained least square (NNLS) deconvolution algorithm; the 
DLS data analysis details are given in Supporting Materials. Although the Cumulant algorithm always assumes monodispersed size distribution, interestingly it fits the $g_{1}(q, t)$ profile (Figure $\left.2 \mathrm{~b}-1\right)$, providing information on the hydrodynamic radius and polydispersity of the particle. The $g_{1}(q, t)$ profile is further fitted in a quantitative manner by the NNLS algorithm, giving information on the mean particle size and distribution (Figure 2c-1,d-1 and f-1). The DLS analysis results are summarized in Table 1.

Table 1. Structural parameters of PS nanoparticles obtained by SAXS, DLS, and TEM analyses.

\begin{tabular}{|c|c|c|c|c|c|c|c|c|}
\hline \multicolumn{9}{|c|}{ PS Nanoparticles } \\
\hline \multirow{2}{*}{$\begin{array}{l}\text { Structure } \\
\text { Parameter }\end{array}$} & \multicolumn{2}{|r|}{ PS-1 } & \multicolumn{2}{|c|}{ PS-2 } & \multicolumn{2}{|c|}{ PS-3 } & \multicolumn{2}{|c|}{ PS-4 } \\
\hline & Water $^{a}$ & Supernatant ${ }^{b}$ & Water & Supernatant & Water & Supernatant & Water & Supernatant \\
\hline \multicolumn{9}{|c|}{ SAXS Analysis } \\
\hline$R_{e}^{c}(\mathrm{~nm})$ & $\begin{array}{c}43.2 \\
(1.0)^{d}\end{array}$ & $\begin{array}{l}43.2 \\
(0.8)\end{array}$ & $\begin{array}{l}29.6 \\
(0.8)\end{array}$ & $\begin{array}{l}29.8 \\
(1.0)\end{array}$ & $\begin{array}{l}14.5 \\
(2.5)\end{array}$ & $\begin{array}{l}16.5 \\
(1.3)\end{array}$ & $\begin{array}{c}8.5 \\
(2.1)\end{array}$ & $\begin{array}{l}12.7 \\
(1.8)\end{array}$ \\
\hline$r_{c}^{e}(\mathrm{~nm})$ & $\begin{array}{l}29.2 \\
(0.6) \\
\end{array}$ & $\begin{array}{l}27.0 \\
(0.6)\end{array}$ & $\begin{array}{l}19.6 \\
(0.5) \\
\end{array}$ & $\begin{array}{l}19.8 \\
(0.5) \\
\end{array}$ & $\begin{array}{c}7.3 \\
(1.8) \\
\end{array}$ & $\begin{array}{c}8.3 \\
(0.6) \\
\end{array}$ & $\begin{array}{c}8.5 \\
(2.1) \\
\end{array}$ & $\begin{array}{l}12.7 \\
(1.8) \\
\end{array}$ \\
\hline$t_{f, c} f(\mathrm{~nm})$ & 5.5 & 6.6 & 2.4 & 3.5 & 1.5 & 2.6 & 1.0 & 1.6 \\
\hline$t_{s} g(\mathrm{~nm})$ & $\begin{array}{l}14.0 \\
(0.8) \\
\end{array}$ & $\begin{array}{l}16.2 \\
(0.5) \\
\end{array}$ & $\begin{array}{l}10.0 \\
(0.6) \\
\end{array}$ & $\begin{array}{l}10.0 \\
(0.9) \\
\end{array}$ & $\begin{array}{c}7.2 \\
(1.8) \\
\end{array}$ & $\begin{array}{c}8.2 \\
(1.1) \\
\end{array}$ & & \\
\hline$t_{f, s}{ }^{h}(\mathrm{~nm})$ & 1.5 & 1.5 & 2.6 & 1.5 & 1.7 & 1.5 & & \\
\hline$\varepsilon^{i}$ & 1.0 & 1.0 & 1.0 & 1.0 & 1.0 & 1.0 & 1.0 & 1.0 \\
\hline$\xi^{j}(\mathrm{~nm})$ & 4.6 & 5.2 & 5.4 & 4.7 & 5.0 & 2.9 & & \\
\hline$R_{\mathrm{g}}{ }^{k}(\mathrm{~nm})$ & 33.5 & 33.5 & 22.9 & 23.1 & 11.2 & 12.9 & 6.6 & 9.8 \\
\hline$R_{\mathrm{g}, \mathrm{IFT}}^{l}(\mathrm{~nm})$ & 33.8 & 33.7 & 23.2 & 23.3 & 12.9 & 13.5 & 8.9 & 10.4 \\
\hline$R_{\max , \mathrm{IFT}}^{m}$ & 45.5 & 45.1 & 30.9 & 31.2 & 16.3 & 18.0 & 10.7 & 14.1 \\
\hline$\underset{(\mathrm{nm})}{D_{\max , \mathrm{IFT}}}{ }^{n}$ & 87.5 & 86.8 & 63.0 & 62.4 & 38.0 & 38.4 & 25.4 & 30.0 \\
\hline$R_{\mathrm{max}, \mathrm{IFT}} / R_{\mathrm{g}, \mathrm{IFT}}$ & 1.35 & 1.34 & 1.33 & 1.34 & 1.26 & 1.33 & 1.20 & 1.36 \\
\hline$D_{\max } / R_{\max , \mathrm{IFT}}$ & 1.92 & 1.92 & 2.04 & 2.00 & 2.33 & 2.13 & 2.37 & 2.13 \\
\hline$R_{\mathrm{g}} / R_{\mathrm{g}, \mathrm{IFT}}$ & 0.99 & 0.99 & 0.99 & 0.99 & 0.87 & 0.96 & 0.74 & 0.94 \\
\hline \multicolumn{9}{|c|}{ DLS Analysis } \\
\hline$R_{\mathrm{h}, \mathrm{z}}{ }^{o}(\mathrm{~nm})$ & 33.5 & & 25.0 & & 14.0 & & 10.4 & \\
\hline$P D I_{\mathrm{DLS}^{p}}(\mathrm{~nm})$ & 0.154 & & 0.055 & & 0.085 & & 0.093 & \\
\hline$\underset{(\mathrm{nm})}{R_{\mathrm{h}, \text { intensity }}{ }^{q}}$ & $\begin{array}{c}39.9 \\
(14.7)\end{array}$ & & $\begin{array}{l}26.9 \\
(7.4)\end{array}$ & & $\begin{array}{l}15.3 \\
(4.7)\end{array}$ & & $\begin{array}{l}11.6 \\
(3.9)\end{array}$ & \\
\hline $\begin{array}{l}R_{\mathrm{h}, \text { voulme }} \\
\quad(\mathrm{nm})\end{array}$ & $\begin{array}{c}28.0 \\
(10.5)\end{array}$ & & $\begin{array}{l}21.9 \\
(6.3)\end{array}$ & & $\begin{array}{l}11.9 \\
(3.7)\end{array}$ & & $\begin{array}{c}8.6 \\
(2.9)\end{array}$ & \\
\hline $\begin{array}{c}R_{\mathrm{h}, \text { number }}{ }^{s} \\
(\mathrm{~nm})\end{array}$ & $\begin{array}{l}21.6 \\
(6.3) \\
\end{array}$ & & $\begin{array}{l}18.6 \\
(4.5) \\
\end{array}$ & & $\begin{array}{c}9.9 \\
(2.5) \\
\end{array}$ & & $\begin{array}{c}7.0 \\
(1.9) \\
\end{array}$ & \\
\hline$R_{\mathrm{h}, \mathrm{z}} / R_{\mathrm{g}}$ & 1.00 & 1.00 & 1.09 & 1.08 & 1.25 & 1.09 & 1.58 & 1.06 \\
\hline$R_{\mathrm{h}, \mathrm{z}} / R_{\mathrm{g}, \mathrm{IFT}}$ & 0.99 & 0.99 & 1.08 & 1.07 & 1.09 & 1.04 & 1.17 & 1.00 \\
\hline$R_{\text {h,intensity }} / R_{e}$ & 0.92 & 0.92 & 0.91 & 0.90 & 1.06 & 0.93 & 1.36 & 0.91 \\
\hline$R_{\mathrm{h}, \text { volume }} / R_{e}$ & 0.65 & 0.65 & 0.74 & 0.73 & 0.82 & 0.72 & 1.01 & 0.68 \\
\hline$R_{\mathrm{g}, \text { number }} / R_{e}$ & 0.50 & 0.50 & 0.63 & 0.62 & 0.68 & 0.60 & 0.82 & 0.55 \\
\hline \multicolumn{9}{|c|}{ TEM Analysis } \\
\hline $\begin{array}{c}R^{t} \\
(\mathrm{~nm})\end{array}$ & $\begin{array}{l}40.5 \\
(1.5)\end{array}$ & & $\begin{array}{l}25.5 \\
(1.5)\end{array}$ & & & & & \\
\hline
\end{tabular}

${ }^{a}$ The scattering from water itself was measured and employed in the data analysis. ${ }^{b}$ The scattering from the supernatant was measured and employed in the data analysis. ${ }^{c}$ Radius of particle in equatorial direction. ${ }^{d}$ Standard deviation. ${ }^{e}$ Radius of particle core. ${ }^{f}$ Thickness of the fuzzy part (interfaced with the shell) of the core. ${ }^{8}$ Thickness of shell part. ${ }^{h}$ Thickness of the fuzzy part (interfaced with water or supernatant) of the shell. ${ }^{i}$ Ellipsoidicity ratio (polar radius/equatorial radius) ${ }^{j}$ Average correlation length of density fluctuation (i.e., random two phases) in the whole particle. ${ }^{k}$ Radius of gyration of particle. ${ }^{l}$ Radius of gyration determined from IFT analysis. ${ }^{m}$ Radius determined from the peak maximum of the $p(r)$ function in IFT analysis. ${ }^{n}$ Maximum dimension determined from the $p(r)$ function in IFT analysis. ${ }^{o} \mathrm{z}$-Averaged hydrodynamic radius. ${ }^{p}$ Polydispersity index of hydrodynamic radius. ${ }^{q}$ Intensity-weighted mean radius. ${ }^{r}$ Volume-weighted mean radius. ${ }^{s}$ Number-weighted mean radius. ${ }^{t}$ Mean radius of particle determined by transmission electron microscopy (TEM) analysis (data from [61]). 

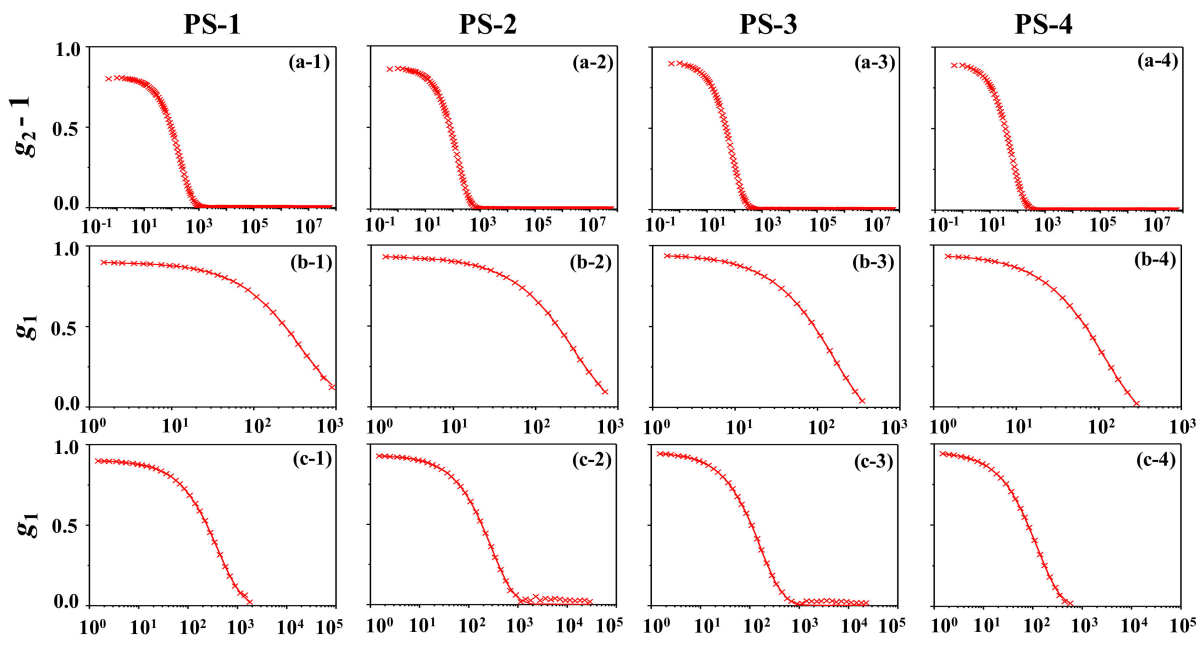

$t(\mu \mathrm{s})$
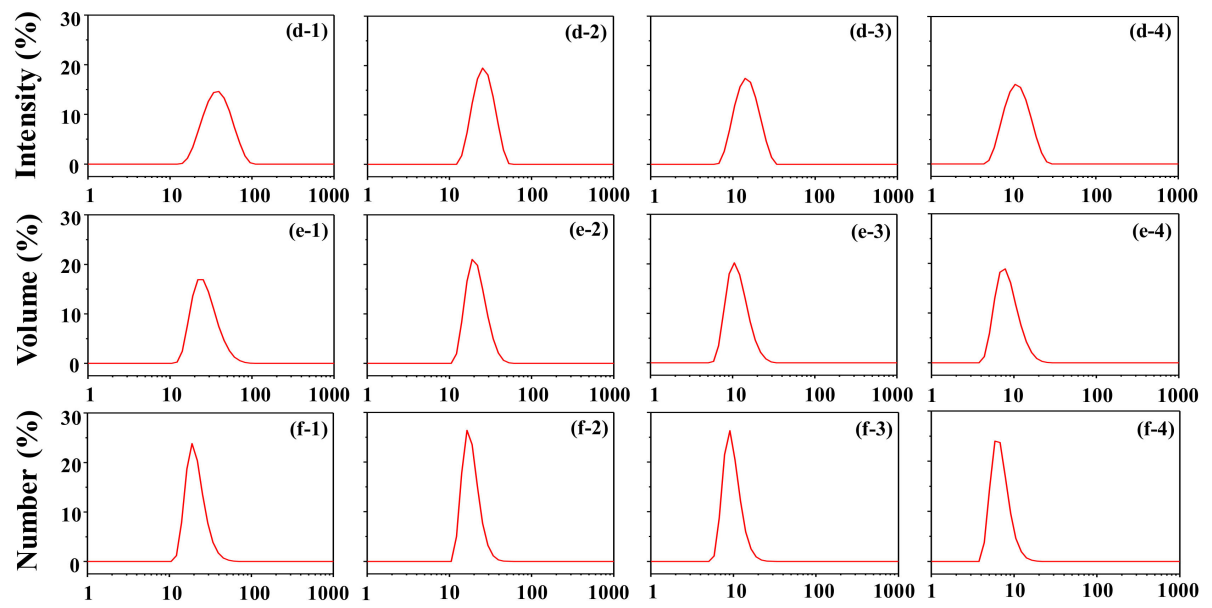

$R_{\mathrm{h}}(\mathrm{nm})$

Figure 2. DLS analyses of PS nanoparticles at $25^{\circ} \mathrm{C}$ : (a-1 to $\left.\mathbf{a}-\mathbf{4}\right)$ autocorrelation profiles measured; (b-1 to b-4) data analysis results, where the symbols are the measured data and the red solid lines were obtained by fitting the data using the cumulant method in the Zetasizer program package; (c-1 to c-4) data analysis results, where the symbols are the measured data and the red solid lines were obtained by fitting the data using the non-negatively constrained least square (NNLS) deconvolution algorithm included in the Zetasizer program package; (d-1 to d-4) intensity-weighted radius distributions obtained by the data analyses; (e) volume-weighted radius distributions obtained from the radius distributions in (d-1 to d-4) and (f) number-weighted radius distributions obtained from the radius distributions in (e-1 to e-4).

The cumulant-based analysis gives a polydispersity $P D I_{\mathrm{DLS}}$ of 0.154 . Taking into account the upper limit $\left(P D I_{D L S}=0.7\right)$ of quality DLS data analysis suggested as a guideline [26], such small $P D I_{D L S}$ value is an indication that the DLS data analysis has been done in a reasonably good quality manner. In addition, the NNLS deconvolution analysis provides a single radius distribution peak. These results collectively inform that PS-1 has a unimodal size distribution. The hydrodynamic radius $R_{\mathrm{h}, \mathrm{z}}$ is $33.5 \mathrm{~nm}$, which is a $z$-averaged radius of gyration of the particle. The mean radius $R_{\mathrm{h}}$ is extracted in three different modes: $R_{\mathrm{h} \text {,intensity }}=39.9 \mathrm{~nm}$ with a standard deviation $\sigma$ of $14.7 \mathrm{~nm}$, which is estimated from the intensity-weighted radius distribution (Figure 2d-1), $R_{\mathrm{h}, \text { volume }}=28.0 \mathrm{~nm}$ with $\sigma=10.5 \mathrm{~nm}$, obtained from the volume-weighted radius distribution (Figure $2 \mathrm{e}-1$ ), and $R_{\mathrm{h}, \text { number }}=21.6 \mathrm{~nm}$ with $\sigma=6.3 \mathrm{~nm}$, determined from the number-weighted radius distribution (Figure 2f-1).

Interestingly, the $R_{\mathrm{h}, \mathrm{z}}$ value is in good agreement with the $R_{\mathrm{g}}$ determined by the quantitative X-ray

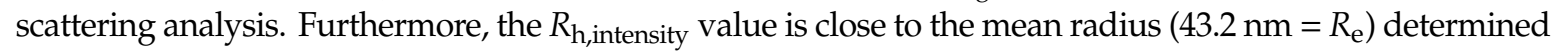
by $\mathrm{X}$-ray scattering analysis and much closer to that $(40.5 \mathrm{~nm})$ estimated by transmission electron microscopy 
(TEM) analysis [61]. However, the $R_{\mathrm{h}, \mathrm{volume}}$ and $R_{\mathrm{h}, \text { number }}$ are significantly smaller than those determined by $X$-ray scattering and TEM analyses; they are $31-35 \%$ and $47-50 \%$ smaller than those determined by $\mathrm{X}$-ray scattering and TEM analyses, respectively. Moreover, the radius distributions are unrealistically 4.2 to 14.7 times larger than those $(1.0$ and $1.5 \mathrm{~nm})$ determined by $X$-ray scattering and TEM analyses.

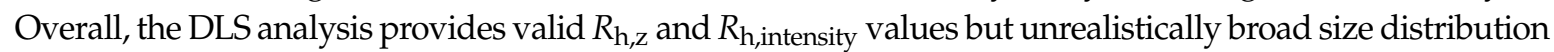
(Figure 3a-1,a-2). The $R_{\mathrm{h}, v o l u m e}$ and $R_{\mathrm{h}, \text { number }}$ are unrealistic and therefore impractical. These unpleasant outputs are attributed to the high uncertainties in the deconvolution of autocorrelation function and very limited resolution of the DLS instrument which may originate from several factors such as (i) much longer wavelength of the used laser source compared to the particle size; (ii) very compact optic geometry; and (iii) only one fixed scattering angle of the detector.

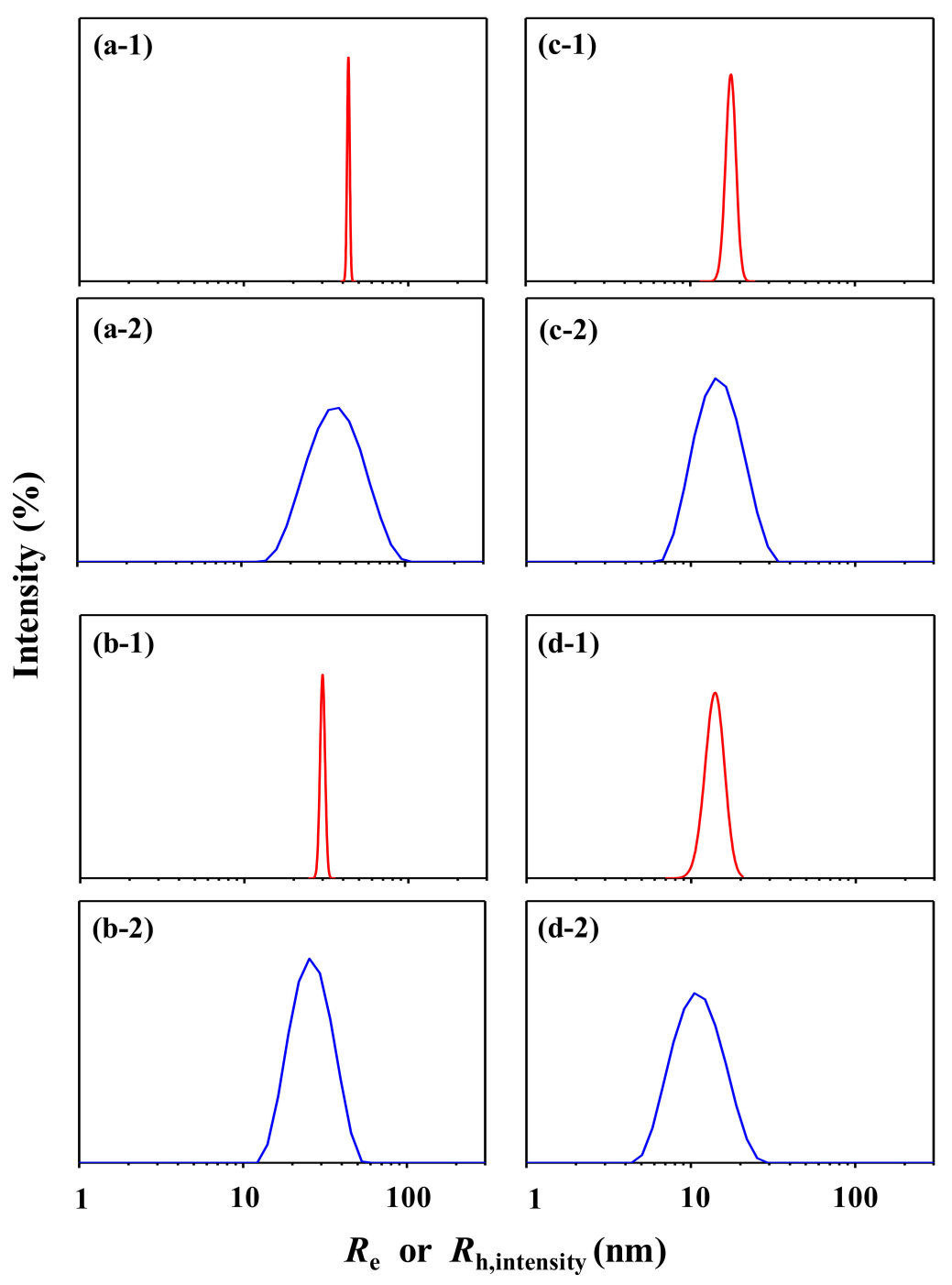

Figure 3. Radius distributions of PS particles determined by X-ray scattering and DLS analyses: (a-1) PS-1 $(\sigma=0.8 \mathrm{~nm})$, from X-ray scattering; (a-2) PS-1 $(\sigma=14.7 \mathrm{~nm})$, from DLS; (b-1) PS-2 $(\sigma=1.0 \mathrm{~nm})$, from X-ray scattering; (b-2) PS-2 $(\sigma=7.4 \mathrm{~nm})$, from DLS; (c-1) PS-3 $(\sigma=1.3 \mathrm{~nm})$, from X-ray scattering; $(\mathbf{c}-2)$ PS-3 $(\sigma=4.7 \mathrm{~nm})$, from DLS; $(\mathbf{d}-1)$ PS-4 $(\sigma=1.8 \mathrm{~nm})$, from X-ray scattering; $(\mathbf{d}-2)$ PS-4 $(\sigma=3.9 \mathrm{~nm})$, from DLS. Here, it is noted that the radius distributions based on the scattering intensities in (a-1), (b-1), (c-1), and (d-1) were obtained from the radius distribution based on the number populations in Figure 1i, Figure 4i, Figure 5i, and Figure $6 \mathrm{i}$ using a relation of the scattering intensity and the volume of the particles in population; such relation is given in Supporting Information. The X-ray scattering analyses were conducted for the scattering data corrected with the supernatant media. 

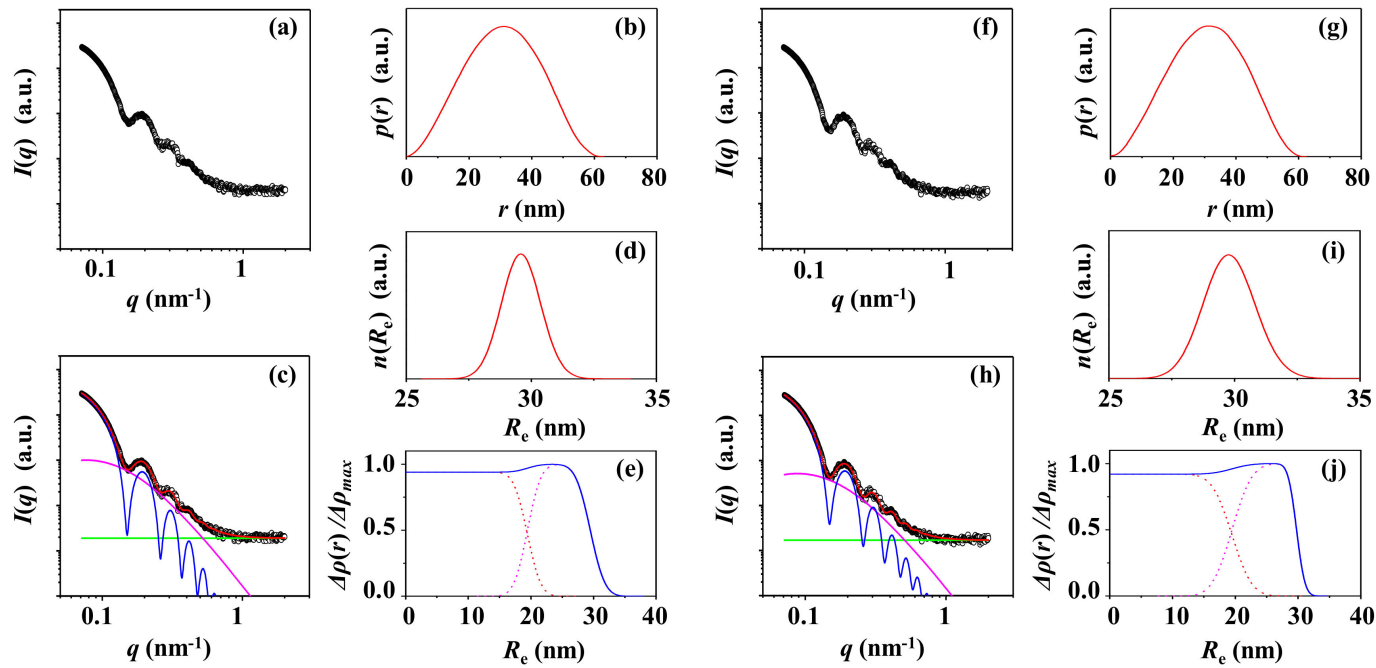

Figure 4. X-ray scattering data analysis of PS-2 particle. (a) SAXS profile measured at room temperature and corrected for water as the solution medium: (b) pair distance distribution functions $p(r)$ obtained from the scattering profile using the IFT method; (c) data analysis results; (d) radius distribution obtained by the data analysis in (c); (e) density distribution obtained by the data analysis in (c). (f) SAXS profile measured at room temperature and corrected for the supernatant as a solution medium: (g) pair distance distribution functions $p(r)$ obtained from the scattering profile using the IFT method; (h) data analysis results; (i) radius distribution obtained by the data analysis in (h); (j) density distribution obtained by the data analysis in (h). (c, h) the open symbols are the measured data and the red solid line represents the sum of the profiles obtained by fitting the data using two-phase ellipsoid model (blue line) and local random two-phase contributions (purple line) and the background (green line).
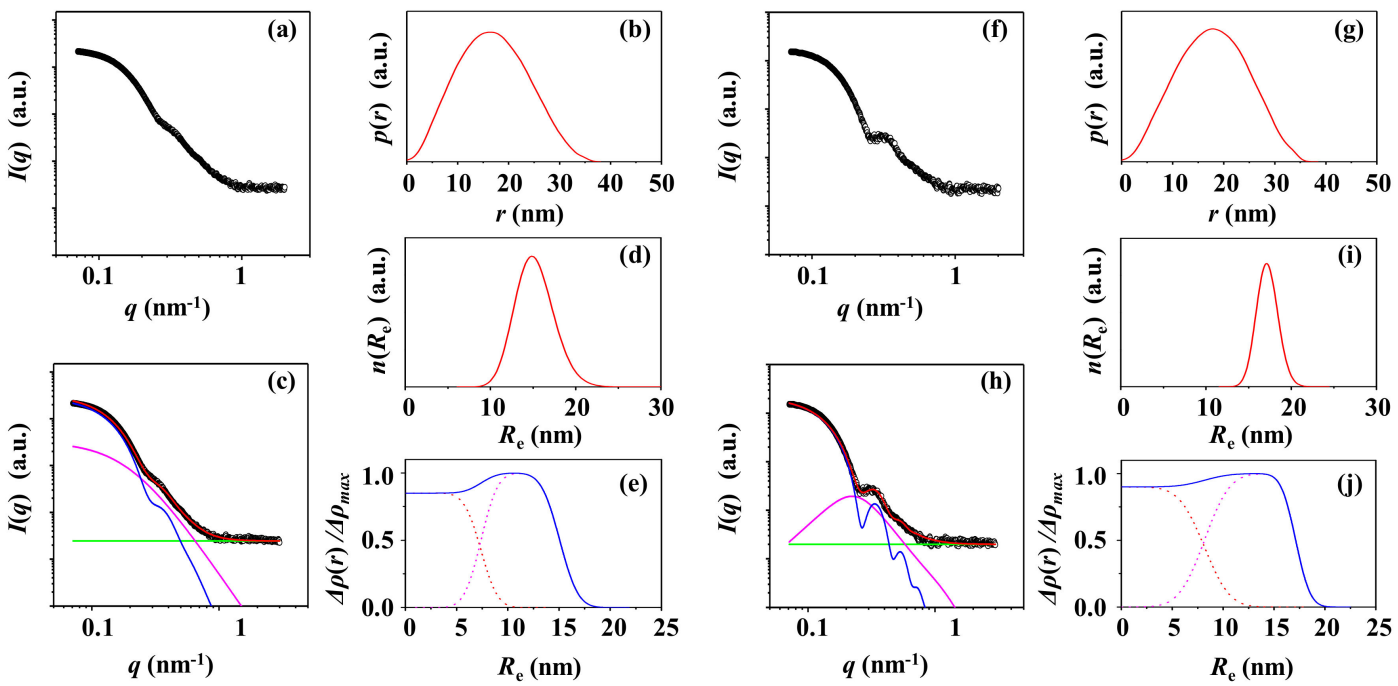

Figure 5. X-ray scattering data analysis of PS-3 particle. (a) SAXS profile measured at room temperature and corrected for water as the solution medium: (b) pair distance distribution functions $p(r)$ obtained from the scattering profile using the IFT method; (c) data analysis results; (d) radius distribution obtained by the data analysis in (c); (e) density distribution obtained by the data analysis in (c). (f) SAXS profile measured at room temperature and corrected for the supernatant as a solution medium: (g) pair distance distribution functions $p(r)$ obtained from the scattering profile using the IFT method; (h) data analysis results; (i) radius distribution obtained by the data analysis in (h); (j) density distribution obtained by the data analysis in (h). (c, h) the open symbols are the measured data and the red solid line represents the sum of the profiles obtained by fitting the data using two-phase ellipsoid model (blue line) and local random two-phase contributions (purple line) and the background (green line). 

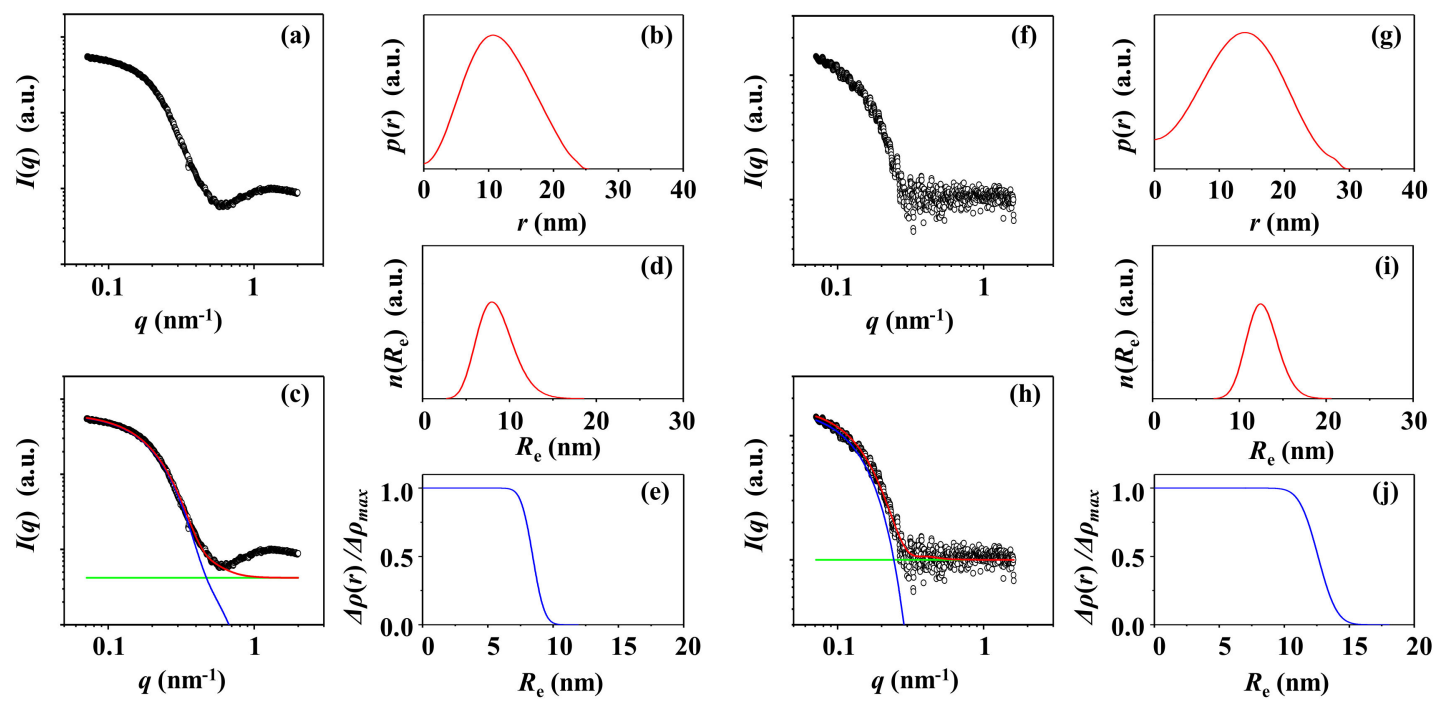

Figure 6. X-ray scattering data analysis of PS-4 particle. (a) SAXS profile measured at room temperature and corrected for water as the solution medium: (b) pair distance distribution functions $p(r)$ obtained from the scattering profile using the IFT method; (c) data analysis results; (d) radius distribution obtained by the data analysis in (c); (e) density distribution obtained by the data analysis in (c). (f) SAXS profile measured at room temperature and corrected for the supernatant as a solution medium: (g) pair distance distribution functions $p(r)$ obtained from the scattering profile using the IFT method; (h) data analysis results; (i) radius distribution obtained by the data analysis in (h); (j) density distribution obtained by the data analysis in $(\mathbf{h})$. $(\mathbf{c}, \mathbf{h})$ the open symbols are the measured data and the red solid line represents the sum of the profiles obtained by fitting the data using two-phase ellipsoid model (blue line) and local random two-phase contributions (purple line) and the background (green line).

\subsection{PS-2}

Figure 4a shows a representative of the synchrotron X-ray scattering data of the PS-2 solution corrected for the water medium. Figure $4 \mathrm{f}$ presents the $\mathrm{X}$-ray scattering data of the PS-2 solution corrected for the supernatant. These scattering data have been successfully analyzed by using both the IFT method and the two-phase ellipsoidal model approach combined with the local density fluctuations based on random two phases. The analysis results are illustrated in Figure $4 b-e, g-j$. The determined structural parameters are listed in Table 1.

From the scattering data corrected with the water medium, the $p(\mathrm{r})$ profile is obtained to be like symmetric bell shape with $D_{\text {max,IFT }} / R_{\text {max,IFT }}=2.04$ and $R_{\text {max,IFT } /} / R_{\mathrm{g}, \mathrm{IFT}}=1.33$, which is close to 1.36 . Together with $R_{\mathrm{g}} / R_{\mathrm{g}, \mathrm{IFT}}=0.98$ and $\varepsilon=1.0$, these results collectively confirm that PS-2 is spherical. The spherical PS-2 particle is characteristic of a set of structural parameters: $R_{\mathrm{g}}=22.9 \mathrm{~nm}, R_{\mathrm{e}}=29.6 \mathrm{~nm}$, $r_{\mathrm{c}}=19.6 \mathrm{~nm}, t_{\mathrm{s}}=10.0 \mathrm{~nm}$, and $\xi=5.4 \mathrm{~nm}$. The $\Delta \rho(r)$ profile has been extracted. Similar shape and structural parameters have been determined from the scattering data corrected for the supernatant, informing that the X-ray scattering data of the PS-2 solution are influenced very little by the surfactant molecules present in the solution.

The DLS data of the PS-2 solution are presented in Figure 2a-2. The $g_{1}(q, t)$ profile is reasonably fitted by the cumulant method as well as by the NNLS algorithm (Figure $2 b-2, c-2$ ). The DLS analysis results are summarized in Table 1 and Figure 2d-2-f-2. $P D I_{D L S}=0.055$, which is lower than that of PS- 1 and much lower than the upper limit (0.7) of quality DLS data analysis. However, $R_{\mathrm{h}, \mathrm{z}}=25.0 \mathrm{~nm}$, that is $9 \%$ larger than the $R_{\mathrm{g}}$ determined by the quantitative $X$-ray scattering analysis. $R_{\mathrm{h}, \text { intensity }}=26.9 \mathrm{~nm}$, which is $9 \%$ smaller than the $R_{\mathrm{e}}$ determined by X-ray scattering analysis, but $5 \%$ larger than the mean radius $(25.5 \mathrm{~nm})$ determined by TEM [61]. $R_{\mathrm{h} \text {,volume }}=21.9 \mathrm{~nm}$ and $R_{\mathrm{h}, \text { number }}=18.6 \mathrm{~nm}$, which are much smaller than the mean radii determined by X-ray scattering and TEM analyses. Depending on the weighted modes (namely, intensity-, volume-, and number-weighted distributions), the $\sigma$ values 
range in 7.4 to 4.5 , which are much larger than those ( 0.8 and 1.5$)$ determined by X-ray scattering and TEM analyses respectively. The radius distributions are unrealistically too broad (Figure 3b-1,b-2). Overall, only $R_{\mathrm{h}, \mathrm{z}}$ and $R_{\mathrm{h}, \text { intensity values are meaningful for PS-2. }}$

\subsection{PS-3}

Figure 5a shows a representative of the X-ray scattering data of the PS-3 solution corrected for the water medium. The $p(r)$ profile is slightly asymmetric; in particular, the part in the high $r$ region is a little bit far from the symmetry (Figure $5 \mathrm{~b}$ ). From the IFT analysis, $R_{\mathrm{g}, \mathrm{IFT}}=12.9 \mathrm{~nm}$

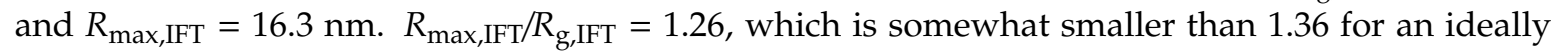
homogenous sphere. $D_{\text {max,IFT }} / R_{\text {max,IFT }}=2.33$, which is larger than 2 for an ideal sphere.

The scattering data have been further analyzed in a quantitative manner. The scattering profile is satisfactorily fitted by using the two-phase ellipsoidal model combined with the local density fluctuations based on random two phases, as shown in Figure $5 \mathrm{c}$. The quantitative analysis results are compared with the IFT analysis results in Table $1 . R_{\mathrm{g}}=11.2 \mathrm{~nm}$, which is $13 \%$ smaller than that determined by the IFT analysis. Other structural parameters obtained are as follows: $R_{\mathrm{e}}=14.5 \mathrm{~nm}$, $r_{\mathrm{c}}=7.3 \mathrm{~nm}, t_{\mathrm{s}}=7.2 \mathrm{~nm}, \xi=5.0 \mathrm{~nm}$, and $\varepsilon=1.0$. In addition, the density profile is determined (Figure 5e). In particular, the $\varepsilon$ value indicates that PS-3 is spherical. PS-3 is found to have a unimodal radius distribution (Figure $5 \mathrm{~d}$ ).

Moreover, the scattering profile has been corrected for the supernatant medium, which is a little bit different from that corrected for the water medium. The scattering data have been analyzed successfully by using the IFT and structural model approaches. The corrected scattering data and analysis results are displayed in Figure $5 \mathrm{f}-\mathrm{j} . R_{\text {max,IFT }} / R_{\mathrm{g}, \mathrm{IFT}}=1.33(=18.0 / 13.5)$, which is much closer to $1.36 ; D_{\text {max }, \mathrm{IFT}} / R_{\max , \mathrm{IFT}}=2.13(=38.4 / 18.0)$, which is close to 2.0. These ratios suggest that the shape of PS-3 is closer to spherical. From the structural model analysis, $R_{\mathrm{g}}=12.9 \mathrm{~nm}, R_{\mathrm{e}}=16.5 \mathrm{~nm}, r_{\mathrm{C}}=8.3 \mathrm{~nm}$, $t_{\mathrm{s}}=8.2 \mathrm{~nm}, \xi=2.9 \mathrm{~nm}$, and $\varepsilon=1.0$. The density profile is determined additionally. Overall, it is again vindicated that PS-3 is spherical and in a unimodal radius distribution.

The DLS data and analysis results are shown in Figure 2a-3-f-3) and Table 1. Briefly, the $P D I_{\mathrm{DLS}}=0.085$, $R_{\mathrm{h}, \mathrm{z}}=14.0 \mathrm{~nm}, R_{\mathrm{h} \text {,intensity }}=15.3 \mathrm{~nm}, R_{\mathrm{h}, \text { volume }}=11.9 \mathrm{~nm}$, and $R_{\mathrm{h}, \text { number }}=9.9 \mathrm{~nm}$. A unimodal radius distribution is found.

The above X-ray scattering and DLS analyses collectively provide key structural features on the PS-3 particle as follows.

First, the X-ray scattering profile corrected for the water medium is slightly different from that corrected for the supernatant medium. This difference is indicative of discernible contributions of the surfactant molecules and their possible assemblies present in the PS-3 solution to the measured X-ray scattering signals.

Second, for the X-ray scattering data corrected from the water medium, the $p(r)$ profile is somewhat asymmetric particularly in the high $r$ region. As a result, the $R_{\max , \mathrm{IFT}} / R_{\mathrm{g}, \mathrm{IFT}}$ and $D_{\mathrm{max}, \mathrm{IFT}} / R_{\mathrm{max}, \mathrm{IFT}}$ values are significantly far from those of a spherical particle. In comparison, for the $\mathrm{X}$-ray scattering data corrected from the supernatant medium, the $p(r)$ profile is more like symmetric; the $R_{\max , I F T} / R_{\mathrm{g}, I F T}$ and

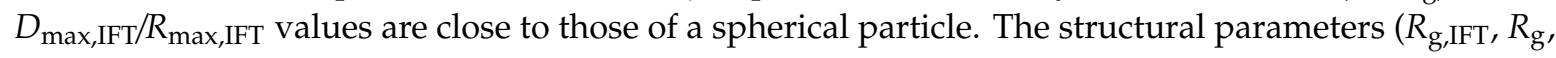
and $R_{\mathrm{e}}$ ) are larger than those determined from the scattering data corrected for the water medium. The radius distribution is much narrower than that obtained from the scattering data corrected for the water medium. These structural differences could be attributed to the surfactant molecules and their possible assemblies present in the PS-3 solution. Namely, in the case of PS-3, the particle dimension $\left(R_{\mathrm{g}}=12.9 \mathrm{~nm}\right.$ and $\left.R_{\mathrm{e}}=16.5 \mathrm{~nm}\right)$ is not large enough to override all scattering signals from the surfactant molecules and their possible assemblies present together in the solution. As discussed above, these kinds of surfactant effects apparently could not be observed for the PS- $1\left(R_{\mathrm{g}}=33.5 \mathrm{~nm}\right.$ and $\left.R_{\mathrm{e}}=43.2 \mathrm{~nm}\right)$ and PS-2 $\left(R_{\mathrm{g}}=23.1 \mathrm{~nm}\right.$ and $\left.R_{\mathrm{e}}=29.8 \mathrm{~nm}\right)$ solutions.

Third, the $R_{\mathrm{h}, \mathrm{z}}$, which is determined by the DLS analysis, is $25 \%$ larger than the $R_{\mathrm{g}}$ determined from the scattering data corrected for the water medium and only $9 \%$ larger than the $R_{\mathrm{g}}$ determined 
from the scattering data corrected for the supernatant medium. The $R_{\mathrm{h} \text {,intensity }}$ value is $7 \%$ larger than $R_{\mathrm{e}}$ determined from the scattering data corrected for the water medium and $6 \%$ smaller than that determined from the scattering data corrected for the supernatant medium. These results collectively suggest that the DLS data are mainly driven from the PS-3 particles but negligibly contributed from the surfactant molecules and their possible assemblies present in the solution. Nevertheless, the particle radius distribution is much broader than that determined by the $\mathrm{X}$-ray scattering analysis. Such the insensitivity to the surfactant molecules as well as the broader radius distribution are caused by the laser source' long wavelength, compact optic geometry, and $90^{\circ}$-fixed scattering angle in the DLS instrument.

Finally, the $R_{\mathrm{h} \text {,volume }}$ and $R_{\mathrm{h} \text {,number }}$ values are much smaller than the $R_{\mathrm{h} \text {,intensity value as well as }}$ the $R_{\mathrm{e}}$ value determined by the $\mathrm{X}$-ray scattering analysis. All radius distributions (intensity-, volume-, and number-weighted size distributions) are too broad unrealistically, compared to that determined by the $\mathrm{X}$-ray scattering analysis (Figure $3 \mathrm{c}-1, \mathrm{c}-2$ ).

\subsection{PS-4}

Figure 6a shows a representative X-ray scattering profile of the PS-4 solution corrected for the water medium. The IFT analysis provides an asymmetric $p(r)$ profile (Figure $6 \mathrm{~b}$ ), $R_{\mathrm{g}, \mathrm{IFT}}=8.9 \mathrm{~nm}$,

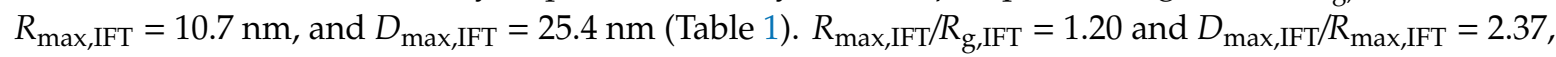
informing that PS-4 behaves a shape far from sphere. The scattering data have been further analyzed in a quantitative manner. The scattering data in the region of $q<0.6 \mathrm{~nm}^{-1}$ are well fitted by using the two phase ellipsoidal model combined with the local density fluctuations based on random two phases; but the scattering data in the region of $q>0.6 \mathrm{~nm}^{-1}$ could be unfitted completely (Figure 6c). From the fitted scattering data part, structural parameters have been extracted: $R_{\mathrm{g}}=6.6 \mathrm{~nm}, R_{\mathrm{e}}=8.5 \mathrm{~nm}, r_{\mathrm{c}}=8.5 \mathrm{~nm}$, $\varepsilon=1.0$, unimodal size distribution, and density profile (Table 1; Figure $6 \mathrm{~d}, \mathrm{e}$ ).

Figure $6 \mathrm{f}$ presents the X-ray scattering data corrected for the supernatant medium. This scattering profile does show no peak over the region of $q>0.6 \mathrm{~nm}^{-1}$, which is different from that corrected for the water medium. The scattering profile has been analyzed by using the IFT method and the two-phase ellipsoidal model combined with the local density fluctuations based on random two phases (Figure $6 \mathrm{~g}, \mathrm{~h}$ ). The IFT analysis gives an inadequate $p(r)$ profile in the low $\mathrm{r}$ region which represents the smaller structural details. It is probably due to the noisy scattering data in the high q region. The structural parameters were obtained: $R_{\mathrm{g}, \mathrm{IFT}}=10.4 \mathrm{~nm}, R_{\max , \mathrm{IFT}}=14.1 \mathrm{~nm}, D_{\text {max,IFT }}=30.0 \mathrm{~nm}$,

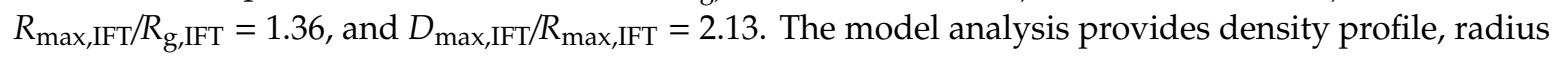
distribution, and a set of parameters: $R_{\mathrm{g}}=9.8 \mathrm{~nm}, R_{\mathrm{e}}=12.7 \mathrm{~nm}$, and $\varepsilon=1.0$ (Figure 6i,j and Table 1). Furthermore, the numerical Fourier transformation was performed on the extrapolated scattering intensity profile from the structural model analysis to obtain the $p(r)$ profiles. All $p(r)$ profiles that were extracted using this method are shown in Supporting Information. The $R_{\mathrm{g}}$ values extracted from the IFT analysis and numerical Fourier transformation are similar.

In addition, the DLS analysis gives radius and radius distributions: $R_{\mathrm{h}, \mathrm{z}}=10.4 \mathrm{~nm}, P D I_{\mathrm{DLS}}=0.093$, $R_{\mathrm{h} \text {,intensity }}=11.6 \mathrm{~nm}, R_{\mathrm{h}, \text { volume }}=8.6 \mathrm{~nm}$, and $R_{\mathrm{h}, \text { number }}=7.0 \mathrm{~nm}$ (Figure 2a-4-f-4). This analysis confirms again that PS-4 exhibits a unimodal radius distribution.

The above X-ray scattering and DLS analyses provide structural feature details on PS-4 below.

First, surprisingly the PS-4 solution shows a broad and weak peak over the region of $q>0.6 \mathrm{~nm}^{-1}$ in the X-ray scattering profile corrected for the water medium, in addition to the scattering signals originated from the PS-4 particle itself. Such broad and weak peak could not be discernible in the X-ray scattering profile corrected for the supernatant medium. For the scattering peak centered at $q=1.3 \mathrm{~nm}^{-1}$, the $d$-spacing is estimated to be $4.8 \mathrm{~nm}$. This $d$-spacing value is much larger than the dimension of the individual surfactant molecules but smaller than the dimension of the PS-4 particle. Taking these facts into account, the scattering peak at $q>0.6 \mathrm{~nm}^{-1}$ may be attributed to assemblies of the surfactant molecules present in the PS- 4 solution. Their size is not small enough, compared to the dimension of PS-4. Therefore, their contributions are severely reflected in the measured scattering profile. 
Second, the structural model analysis confirms that PS-4 is a single-phase spherical particle rather than a two-phase particle. This result may be an evidence that the PS-4 particles were prepared in a relatively small scale and thus could not have enough opportunity to be grown as two-phase particle during their emulsion polymerization process.

Third, for the X-ray scattering profile corrected with the water medium, its $p(r)$ profile shows asymmetric characteristics. Such characteristics are directly reflected in $R_{\max , \mathrm{IFT}} / R_{\mathrm{g}, \mathrm{IFT}}$ and $D_{\text {max,IFT }} / R_{\text {max,IFT }}$; these ratios are deviated from 1.36 and 2.00 , respectively. These results collectively support the presence of the surfactant molecules and their assemblies in the PS-4 solution.

Fourth, the $R_{\mathrm{h}, \mathrm{z}}$ obtained by the DLS analysis is $58 \%$ larger than the $R_{\mathrm{g}}$ determined from the scattering data corrected for the water medium but only $6 \%$ larger than the $R_{\mathrm{g}}$ determined from the scattering data corrected for the supernatant medium. The $R_{\mathrm{h} \text {,intensity }}$ value is $36 \%$ larger than the $R_{\mathrm{e}}$ determined from the scattering data corrected for the water medium. However, the value is $9 \%$ smaller than that determined from the scattering data corrected for the supernatant medium. These results confirm again that the surfactant molecules employed in the PS-4 particle synthesis remain in the particle suspension. Moreover, these comparisons suggest that the DLS data are mainly driven from the PS-4 particles, but surprisingly insensitive even to the presence of the surfactant assemblies revealing a $d$-spacing of $4.9 \mathrm{~nm}$ in the X-ray scattering in addition to the surfactant molecules. Such insensitivities to the existing surfactants and their assemblies are evidence for the poor limits of the DLS instrument, consequently causing errors in determining particle size and distribution.

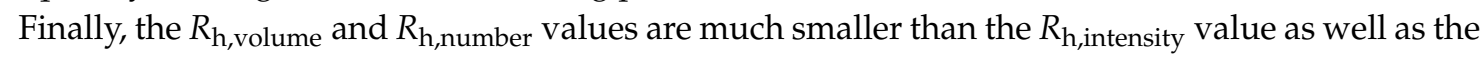
$R_{\mathrm{e}}$ value determined by the $\mathrm{X}$-ray scattering analysis. Furthermore, the radius distributions estimated by the DLS analysis are always too broad, compared to that determined by the $\mathrm{X}$-ray scattering analysis (Figure $3 \mathrm{~d}-1, \mathrm{~d}-2$ ). Therefore, the $R_{\mathrm{h}, \text { volume }}, R_{\mathrm{h} \text {,number }}$ and radius distributions provided by the DLS analysis are meaningless practically.

\section{Conclusions}

In this study, a series of PS nanoparticles, which were prepared by conventional surfactant-assisted emulsion polymerization, has been investigated in terms of morphological structure and size distribution.

The quantitative X-ray scattering analysis has been successfully performed on the PS particles in aqueous media, providing key features as follows. The PS solution individuals are found of (i) spherical particles in very narrow unimodal size distribution and (ii) surfactant molecules and their assemblies. The PS-1, PS-2, and PS-3 particle individuals are characteristic of revealing two-phase (core and shell) nanoparticles, whereas PS-4 is a single-phase nanoparticle; they all exhibit unique electron density profiles. For the PS particle individuals, structural parameter details have been determined in high precision and accuracy. The particle size $\left(R_{\mathrm{g}}\right.$ and $\left.R_{\mathrm{e}}\right)$ is in the decreasing order: PS-1 $>$ PS-2 $>$ PS-3 > PS-4; in contrast, the broadening of size distribution is in the increasing order: PS- $1<$ PS- $2<$ PS-3 $<$ PS-4.

The PS solutions have been further examined by DLS analysis. All analyses have done with very low $P D I_{\text {DLS }}$ values $(0.055-0.154)$, suggesting that all PS particles are spherical. Fortunately, due to the spherical nature, the particle size ( $R_{\mathrm{h}}$ and $\left.R_{\mathrm{h} \text {,intensity }}\right)$ of each particle system is determined to be reasonably close to those $\left(R_{\mathrm{g}}\right.$ and $\left.R_{\mathrm{e}}\right)$ extracted from the $\mathrm{X}$-ray scattering and TEM analysis. All PS particle systems are confirmed to behave unimodal size distributions. Nevertheless, the determined size distributions are too broad unrealistically, compared to those extracted by $\mathrm{X}$-ray scattering analysis. In contrast, the $R_{\mathrm{h} \text {,volume }}$ and $R_{\mathrm{h}, \text { number values are always too small unrealistically. Furthermore, the DLS }}$ analysis are completely insensitive to the surfactant molecules and their assemblies present in the particle solutions. Overall, the compact and fixed-angle DLS instrument of this study is confirmed to have severe resolution limits and thus applicable to measure $R_{\mathrm{h}}$ and $R_{\mathrm{h} \text {,intensity }}$ for only spherical particles; the $R_{\mathrm{h} \text {,volume, }}, R_{\mathrm{h} \text {,number, }}$ and all different mode based size distributions could give no meaningful information even though they were extracted from the spherical particles. 
In summary, the quantitative X-ray scattering analysis together with qualitative DLS analysis has confirmed that the individual PS particle systems were successfully prepared with spherical shape in a very narrow unimodal size distribution. The X-ray scattering analysis has further provided morphological structure details (shape, dimension, density profile, size, and size distribution) of all PS nanoparticles.

Supplementary Materials: The following are available online at http://www.mdpi.com/2073-4360/12/2/477/s1, Figure S1: Pair distance distribution functions $p(r)$ obtained from numerical Fourier transformation of the extrapolated scattering intensity profile from model analysis, Table S1: Structural parameters of PS nanoparticles determined from $p(r)$ function obtained by numerical Fourier transformation of respective extrapolated scattering intensity profile acquired in the quantitative structural model analysis.

Author Contributions: C.H.C., K.S.J., and M.R. designed and initiated the research. J.C.W. prepared nanoparticle solutions and conducted synchrotron X-ray scattering and DLS measurements. J.C.W. analyzed DLS data. J.C.W., L.X., and K.H.N. analyzed X-ray scattering data. J.C.W. prepared a draft of manuscript. All authors contributed to discussions on the data analyses and results and to the finalization of manuscript. All authors have read and agreed to the published version of the manuscript.

Funding: This research was funded by National Research Foundation of Korea: 2017R1C1B1004449.

Acknowledgments: K.S.J. gratefully acknowledges financial support from the National Research Foundation of Korea (NRF) (grant no. 2017R1C1B1004449).

Conflicts of Interest: The authors declare no conflict of interest.

\section{References}

1. Gilbert, R.G. Emulsion Polymerization, A Mechanistic Approach; Academic Press: London, UK, 1995.

2. Antonietti, M.; Forster, S. Vesicles and liposomes: A self-assembly principle beyond lipids. Adv. Mater. 2003, 15, 1323-1333. [CrossRef]

3. Atanase, L.I.; Riess, G. Self-assembly of block and graft copolymers in organic solvents: An overview of recent advances. Polymers 2018, 10, 62. [CrossRef] [PubMed]

4. Ree, B.J.; Satoh, Y.; Jin, K.S.; Isono, T.; Kim, W.J.; Kakuchi, T.; Satoh, T.; Ree, M. Well-defined stable nanomicelles self-assembled by cyclic and tadpole polymers: A versatile smart carrier platform. NPG Asia Mater. 2017, 9, e453. [CrossRef]

5. Atanase, L.I.; Desbrieres, J.; Riess, G. Micellization of synthetic and polysaccharides-based graft copolymers in aqueous media. Prog. Polym. Sci. 2017, 73, 32-60. [CrossRef]

6. Kim, Y.Y.; Hwang, B.; Song, S.; Ree, B.J.; Kim, Y.; Cho, S.Y.; Heo, K.; Kwon, Y.K.; Ree, M. Well-defined hollow nanochanneled-silica nanospheres prepared with the aid of sacrificial copolymer nanospheres and surfactant nanocylinders. Nanoscale 2015, 7, 14774-14785. [CrossRef]

7. Liu, W.T. Nanoparticles and their biological and environmental applications. J. Biosci. Bioeng. 2006, 102, 1-7. [CrossRef]

8. Zhang, Q.F.; Uchaker, E.; Candelaria, S.L.; Cao, G.Z. Nanomaterials for energy conversion and storage. Chem. Soc. Rev. 2013, 42, 3127-3171. [CrossRef]

9. Patri, A.K.; Dobrovolskaia, M.A.; Stern, S.T.; McNeil, S.E. Chapter 7. Preclinical Characterization of Engineered Nanoparticles Intended for Cancer Therapeutics. In Nanotechnology for Cancer Therapy; Amiji, M.M., Ed.; Taylor Francis Group: Boca Raton, FL, USA, 2006.

10. Siralertmukul, K.; Watcharamul, S.; Wicheanpaisan, N.; Nuisin, R. Potential antibacterial activity of polystyrene nanoparticles/chitosan coated on cotton fabrics. Macromol. Symp. 2015, 354, 324-333. [CrossRef]

11. Gao, Y.; Shi, Y. Design of a single nanoparticle trapping device based on bow-tie-shaped photonic crystal nanobeam cavities. IEEE Photonics J. 2019, 11, 4500408. [CrossRef]

12. Taguchi, M.; Ogami, T.; Ando, J.; Fukuda, T.; Emoto, A. Ag-coated submicron particles of polystyrene formed by dewetting process and their application in multi-functional biosensor-chips. Colloids Surf. A 2018, 558, 171-178. [CrossRef]

13. Lehner, R.; Weder, C.; Petri-Fink, A.; Rothen-Rutishauser, B. Emergence of nanoplastic in the environment and possible impact on human health. Environ. Sci. Technol. 2019, 53, 1748-1765. [CrossRef] [PubMed]

14. Yang, Q.; Li, L.; Zhao, F.; Han, H.; Wang, W.; Tian, Y.; Wang, Y.; Ye, Z.; Guo, X. Hollow silica-polyelectrolyte composite nanoparticles for controlled drug delivery. J. Mater. Sci. 2018, 54, 2552-2565. [CrossRef] 
15. Ehmann, F.; Sakai-Kato, K.; Duncan, R.; Hernán Pérez dela Ossa, D.; Pita, R.; Vidal, J.M.; Kohli, A.; Tothfalusi, L.; Sanh, A.; Tinton, S.; et al. Next-generation nanomedicines and nanosimilars: EU regulators' initiatives relating to the development and evaluation of nanomedicines. Nanomedicine 2013, 8, 849-856. [CrossRef] [PubMed]

16. Chithrani, B.D.; Ghazani, A.A.; Chan, W.C. Determining the size and shape dependence of gold nanoparticle uptake into mammalian cells. Nano Lett. 2006, 6, 662-668. [CrossRef]

17. Cabral, H.; Matsumoto, Y.; Mizuno, K.; Chen, Q.; Murakami, M.; Kimura, M.; Terada, Y.; Kano, M.R.; Miyazono, K.; Uesaka, M.; et al. Accumulation of sub-100 nm polymeric micelles in poorly permeable tumours depends on size. Nat. Nanotechnol. 2011, 6, 815-823. [CrossRef]

18. Döge, N.; Hadam, S.; Volz, P.; Wolf, A.; Schönborn, K.-H.; Blume-Peytavi, U.; Alexiev, U.; Vogt, A. Identification of polystyrene nanoparticle penetration across intact skin barrier as rare event at sites of focal particle aggregations. J. Biophotonics. 2018, 11, e201700169. [CrossRef]

19. Rothbauer, M.; Patel, N.; Gondola, H.; Siwetz, M.; Huppertz, B.; Ertl, P. A comparative study of five physiological key parameters between four different human trophoblast-derived cell lines. Sci. Rep. 2017, 7, 5892. [CrossRef]

20. Gaumet, M.; Vargas, A.; Gurny, R.; Delie, F. Nanoparticles for drug delivery: The need for precision in reporting particle size parameters. Eur. J. Pharm. Biopharm. 2008, 69, 1-9. [CrossRef]

21. Marques-Santos, L.F.; Grassi, G.; Bergami, E.; Faleri, C.; Balbi, T.; Salis, A.; Damonte, G.; Canesi, L.; Corsi, I. Cationic polystyrene nanoparticle and the sea urchin immune system: Biocorona formation, cell toxicity, and multixenobiotic resistance phenotype. Nanotoxicology 2018, 12, 847-867. [CrossRef]

22. Liu, Y.; MarkWorden, R. Size dependent disruption of tethered lipid bilayers by functionalized polystyrene nanoparticles. Biochim. Biophys. Acta 2015, 1848, 67-75. [CrossRef]

23. Cai, X.J.; Woods, A.; Mesquida, P.; Jones, S.A. Assessing the potential for drug-nanoparticle surface interactions to improve drug penetration into the skin. Mol. Pharm. 2016, 13, 1375-1384. [CrossRef] [PubMed]

24. McCarthy, J.; Gong, X.; Nahirney, D.; Duszyk, M.; Radomski, M.W. Polystyrene nanoparticles activate ion transport in human airway epithelial cells. Internatl. J. Nanomed. 2011, 6, 1343-1356. [CrossRef] [PubMed]

25. Brun, N.R.; Beenakker, M.M.T.; Hunting, E.R.; Ebert, D.; Vijver, M.G. Brood pouch-mediated polystyrene nanoparticle uptake during Daphnia magna embryogenesis. Nanotoxicology 2017, 11, 1059-1069. [CrossRef] [PubMed]

26. Stetefeld, J.; McKenna, S.A.; Patel, T.R. Dynamic light scattering: A practical guide and applications in biomedical sciences. Biophys. Rev. 2016, 8, 409-427. [CrossRef]

27. Kestens, V.; Roebben, G.; Herrmann, J.; Jämting, Å.; Coleman, V.; Minelli, C.; Clifford, C.; De Temmerman, P.J.; Mast, J.; Junjie, L.; et al. Challenges in the size analysis of a silica nanoparticle mixture as candidate certified reference material. J. Nanopart. Res. 2016, 18, 171. [CrossRef]

28. Hoo, C.M.; Starostin, N.; West, P.; Mecartney, M.L. A comparison of atomic force microscopy (AFM) and dynamic light scattering (DLS) methods to characterize nanoparticle size distributions. J. Nanopart. Res. 2008, 10, 89-96. [CrossRef]

29. Frisken, B.J. Revisiting the method of cumulants for the analysis of dynamic light-scattering data. Appl. Opt. 2001, 40, 4087-4091. [CrossRef]

30. Patty, P.J.; Frisken, B.J. Direct determination of the number-weighted mean radius and polydispersity from dynamic light-scattering data. Appl. Opt. 2006, 45, 2209-2216. [CrossRef]

31. Xu, Y.; Nakane, N.; Maurer-Spurej, E. Novel test for microparticles in platelet-rich plasma and platelet concentrates using dynamic light scattering. Transfusion 2011, 51, 363-370. [CrossRef]

32. Ree, B.J.; Lee, J.; Satoh, Y.; Kwon, K.; Isono, T.; Satoh, T.; Ree, M. A Comparative study of dynamic light and X-ray scatterings on micelles of topological polymer amphiphiles. Polymers 2018, 10, 1347. [CrossRef]

33. Ree, B.J.; Satoh, T.; Yamamoto, T. Micelle structure details and stabilities of cyclic block copolymer amphiphile and its linear analogues. Polymers 2019, 11, 163. [CrossRef] [PubMed]

34. Lee, J.; Xiang, L.; Byambabaatar, S.; Kim, H.; Jin, K.S.; Ree, M. Bacillus licheniformis $\alpha$-amylase: Structural feature in a biomimetic solution and structural changes in extrinsic conditions. Int. J. Biol. Macromol. 2019, 127, 286-296. [CrossRef] [PubMed] 
35. Bootz, A.; Vogel, V.; Schubert, D.; Kreuter, J. Comparison of scanning electron microscopy, dynamic light scattering and analytical ultracentrifugation for the sizing of poly(butyl cyanoacrylate) nanoparticles. Eur. J. Pharm. Biopharm. 2004, 57, 369-375. [CrossRef]

36. Zhang, J.; Li, Y.; An, F.F.; Zhang, X.; Chen, X.; Lee, C.S. Preparation and size control of sub-100 nm pure nanodrugs. Nano Lett. 2015, 15, 313-318. [CrossRef]

37. Kuntsche, J.; Horst, J.C.; Bunjes, H. Cryogenic transmission electron microscopy (cryo-TEM) for studying the morphology of colloidal drug delivery systems. Int. J. Pharm. 2011, 417, 120-137. [CrossRef] [PubMed]

38. Yuana, Y.; Oosterkamp, T.H.; Bahatyrova, S.; Ashcroft, B.; Garcia, R.P.; Bertina, R.M.; Osanto, S. Atomic force microscopy: A novel approach to the detection of nanosized blood microparticles. J. Thromb. Haemost. 2010, 8, 315-323. [CrossRef]

39. Wolfgang, H.-G.; Dorothee, H.; Klaus-Peter, J.; Carl Georg, F.; Harald, B. Current limitations of SEM and AFM metrology for the characterization of 3D nanostructures. Meas. Sci. Technol. 2011, 22, 094003.

40. Baalousha, M.; Lead, J.R. Rationalizing nanomaterial sizes measured by atomic force microscopy, flow field-flow fractionation, and dynamic light scattering: Sample preparation, polydispersity, and particle structure. Environ. Sci. Technol. 2012, 46, 6134-6142. [CrossRef]

41. Takechi-Haraya, Y.; Goda, Y.; Sakai-Kato, K. Imaging and size measurement of nanoparticles in aqueous medium by use of atomic force microscopy. Anal. Bioanal. Chem. 2018, 410, 1525-1531. [CrossRef]

42. Ballauff, M. Analysis of polymer colloids by small-angle X-ray and neutron scattering: Contrast variation. Adv. Eng. Mater. 2011, 13, 793-802. [CrossRef]

43. Teulon, J.-M.; Godon, C.; Chantalat, L.; Moriscot, C.; Cambedouzou, J.; Odorico, M.; Ravaux, J.; Podor, R.; Gerdil, A.; Habert, A.; et al. On the operational aspects of measuring nanoparticle sizes. Nanomaterials 2019, 9, 18. [CrossRef] [PubMed]

44. Kim, M.; Rho, Y.; Jin, K.S.; Ahn, B.; Jung, S.; Kim, H.; Ree, M. pH-dependent structures of ferritin and apoferritin in solution: Disassembly and reassembly. Biomacromolecules 2011, 12, 1629-1640. [CrossRef]

45. Shin, S.R.; Jin, K.S.; Lee, C.K.; Kim, S.I.; Spinks, G.M.; So, I.; Jeon, J.-H.; Kang, T.M.; Mun, J.Y.; Han, S.-S.; et al. Fullerene attachment enhances performance of a DNA nanomachine. Adv. Mater. 2009, 21, 1907-1910. [CrossRef]

46. Castorph, S.; Riedel, D.; Arleth, L.; Sztucki, M.; Jahn, R.; Holt, M.; Salditt, T. Structure parameters of synaptic vesicles quantified by small-angle x-ray scattering. Biophys. J. 2010, 98, 1200-1208. [CrossRef] [PubMed]

47. Zhao, Y.; Liu, S.; Elsworth, D.; Jiang, Y.; Zhu, J. Pore Structure Characterization of Coal by Synchrotron Small-Angle X-ray Scattering and Transmission Electron Microscopy. Energy Fuels 2014, 28, 3704-3711. [CrossRef]

48. Cervellino, A.; Giannini, C.; Guagliardi, A.; Zanchet, D. Quantitative analysis of gold nanoparticles from synchrotron data by means of least-squares techniques: Least-squares analysis of gold nanoparticles. Eur. Phys. J. B 2004, 41, 485-493. [CrossRef]

49. Hassellov, M.; Readman, J.W.; Ranville, J.F.; Tiede, K. Nanoparticle analysis and characterization methodologies in environmental risk assessment of engineered nanoparticles. Ecotoxicology 2008, 17, 344-361. [CrossRef]

50. Mahl, D.; Diendorf, J.; Meyer-Zaika, W.; Epple, M. Possibilities and limitations of different analytical methods for the size determination of a bimodal dispersion of metallic nanoparticles. Colloids Surf. A Physicochem. Eng. Asp. 2011, 377, 386-392. [CrossRef]

51. Eaton, P.; Quaresma, P.; Soares, C.; Neves, C.; De Almeida, M.P.; Pereira, E.; West, P. A direct comparison of experimental methods to measure dimensions of synthetic nanoparticles. Ultramicroscopy 2017, 182, 179-190. [CrossRef]

52. DeBlois, R.W.; Bean, C.P. Counting and sizing of submicron particles by the resistive pulse technique. Rev. Sci. Instrum. 1970, 41, 909-916. [CrossRef]

53. Pabst, W.; Gregorová, E. Characterization of Particles and Particle Systems; Institute of Chemical Technology (ICT): Prague, Czech, 2007.

54. Braun, A.; Couteau, O.; Franks, K.; Kestens, V.; Roebben, G.; Lamberty, A.; Linsinger, T.P.J. Validation of dynamic light scattering and centrifugal liquid sedimentation methods for nanoparticle characterisation. Adv. Powder Technol. 2011, 22, 766-770. [CrossRef] 
55. Boyd, R.D.; Pichaimuthu, S.K.; Cuenat, A. New approach to intertechnique comparisons for nanoparticle size measurements; using atomic force microscopy, nanoparticle tracking analysis and dynamic light scattering. Colloids Surf. A Physicochem. Eng. Asp. 2011, 387, 35-42. [CrossRef]

56. Kato, H.; Nakamura, A.; Takahashi, K.; Kinugasa, S. Accurate size and size-distribution determination of polystyrene latex nanoparticles in aqueous medium using dynamic light scattering and asymmetrical flow field flow fractionation with multi-angle light scattering. Nanomaterilas 2012, 2, 15-30. [CrossRef] [PubMed]

57. Kim, K.; Kim, J.; Yun, Y.; Ahn, H.; Min, B.; Kim, N.; Rah, S.; Kim, H.; Lee, C.; Seo, I.; et al. Small-angle X-ray Scattering Beamline BL4C SAXS at Pohang Light Source II. Biodesign 2017, 5, 24-29.

58. Glatter, O.J. A new method for the evaluation of small-angle scattering data. J. Appl. Crystallogr. 1977, 10, 415-421. [CrossRef]

59. Takeshita, H.; Poovarodom, M.; Kiya, T.; Arai, F.; Takenaka, K.; Miya, M.; Shiomi, T. Crystallization behavior and chain folding manner of cyclic, star and linear poly(tetrahydrofuran)s. Polymer 2012, 53, 5375-5384. [CrossRef]

60. Li, T.; Senesi, A.J.; Lee, B. Small Angle X-ray Scattering for Nanoparticle Research. Chem. Rev. 2016, 116, 11128-11180. [CrossRef]

61. Hashemi, S. TEM Data from Thermo Fisher Particle Technology; Thermo Fisher Inc.: Waltham, MA, USA, 2017.

62. Siegert, A.J.F. On the fluctuations in signals returned by many independently moving scatterers. In Massachusetts Institute of Technology; Radiation Laboratory Report No. 465; MIT: Cambridge, MA, USA, 1949.

63. Simons, J. The Siegert method in resonance scattering: Relation to L2 methods. Int. J. Quantum Chem. 1981, 20, 779-780. [CrossRef]

(C) 2020 by the authors. Licensee MDPI, Basel, Switzerland. This article is an open access article distributed under the terms and conditions of the Creative Commons Attribution (CC BY) license (http://creativecommons.org/licenses/by/4.0/). 